\title{
Ausgangslage: Anforderung der Digitalisierung
}

Durch eine fortschreitende Digitalisierung vollzieht sich zurzeit eine tiefgreifende Veränderung in unserer Gesellschaft. Wir erleben einen technologischen Wandel hin zur Informationsgesellschaft. Der hiermit einhergehende Strukturwechsel führt zu einer Durchdringung aller Lebensbereiche mit Informations- und Kommunikationstechnik. Die Digitalisierung verändert vielfältige Lebensbereiche und beeinflusst auch das Wirtschaftsleben. Sie ist Basis für neue „digitale Geschäftsmodelle" und ihre wirtschaftliche Bedeutung wird weiter zunehmen. Bekannte Geschäftsmodelle werden durch internetbasierte Dienste verdrängt. Um im globalen Wettbewerb bestehen zu können, sind disruptive Geschäftsmodelle schnell zu adaptieren. Disruptive Technologien haben nicht nur Einfluss auf die Technologie selbst, sondern sie verändern auch den Menschen und sein Verhalten.

Ohne den Einsatz von Informationstechnik kann ein Unternehmen immer weniger im Wettbewerb erfolgreich existieren. Auch bei der Modernisierung der öffentlichen Verwaltung spielt die Informationstechnologie eine zunehmend wichtiger werdende Rolle. Auf dem Weg zur Informationsgesellschaft nimmt die Qualität und Quantität der Information immer weiter zu und der Geschäftserfolg wird immer abhängiger von der Bereitstellung von Informationen. Datenschutz und Informationssicherheit spielen bei der Digitalisierung eine bedeutende Rolle. Die fehlende Verfügbarkeit der Informations- und Kommunikationstechnik, der unkontrollierte Abfluss und die Manipulation von Daten können für ein Unternehmen, für Behörden oder den Bürger ${ }^{1}$ schwere Schäden nach sich ziehen.

\footnotetext{
${ }^{1}$ Im Sinne einer besseren Lesbarkeit wird in diesem Dokument stets die männliche Form verwendet und gilt für beide Geschlechter.
} 